\title{
Progressive Periodontal Disease and Risk of Very Preterm Delivery
}

\author{
Steven Offenbacher, DDS, PhD, Kim A. Boggess, MD, Amy P. Murtha, MD, Heather L. Jared, MS, \\ Susan Lieff, PhD, Rosemary G. McKaig, PhD, Sally M. Mauriello, Kevin L. Moss, and James D. Beck, PhD
}

OBJECTIVE: The goal was to estimate whether maternal periodontal disease was predictive of preterm (less than 37 weeks) or very preterm (less than 32 weeks) births.

METHODS: A prospective study of obstetric outcomes, entitled Oral Conditions and Pregnancy (OCAP), was conducted with 1,020 pregnant women who received both an antepartum and postpartum periodontal examination. Predictive models were developed to estimate whether maternal exposure to either periodontal disease at enrollment (less than 26 weeks) and/or periodontal disease progression during pregnancy, as determined by comparing postpartum with antepartum status, were predictive of preterm or very preterm births, adjusting for risk factors including previous preterm delivery, race, smoking, social domain variables, and other infections.

RESULTS: Incidence of preterm birth was $11.2 \%$ among periodontally healthy women, compared with $28.6 \%$ in women with moderate-severe periodontal disease (adjusted risk ratio [RR] 1.6, 95\% confidence interval [CI] 1.1-2.3). Antepartum moderate-severe periodontal disease was associated with an increased incidence of spontaneous preterm births $(15.2 \%$ versus $24.9 \%$, adjusted RR 2.0, 95\% CI 1.2-3.2). Similarly, the unadjusted rate of very preterm delivery was $6.4 \%$ among women with periodontal disease progression, significantly higher than the $1.8 \%$ rate among women without disease progression (adjusted RR 2.4, 95\% CI 1.1-5.2).

From the Departments of Periodontology and Dental Ecology, Center for Oral and Systemic Diseases, University of North Carolina School of Dentistry, Chapel Hill, North Carolina; Department of Obstetrics and Gynecology, University of North Carolina, Chapel Hill, North Carolina; Department of Obstetrics and Gynecology, Duke University, Durham, North Carolina; and National Institutes of Allergy and Infectious Diseases, Bethesda, Maryland.

This study was supported by the following grants: RO1-DE-12453, P-60-DE13079, T-32-DE-07310, M01-RR-00046.

Corresponding author: Steven Offenbacher, DDS, PhD, MMSc, OraPharma Distinguished Professor of Periodontal Medicine, Director, Center for Oral and Systemic Diseases, UNC School of Dentistry, CB \#7455, DRC Room 222, University of North Carolina at Chapel Hill, Chapel Hill, NC 27599-7455; e-mail: steve_offenbacher@dentistry.unc.edu.

(C) 2005 by The American College of Obstetricians and Gynecologists. Published by Lippincott Williams \& Wilkins.

ISSN: 0029-7844/05
CONCLUSION: The OCAP study demonstrates that maternal periodontal disease increases relative risk for preterm or spontaneous preterm births. Furthermore, periodontal disease progression during pregnancy was a predictor of the more severe adverse pregnancy outcome of very preterm birth, independently of traditional obstetric, periodontal, and social domain risk factors. (Obstet Gynecol 2006;107:29-36)

\section{LEVEL OF EVIDENCE: II-2}

$\mathrm{R}$ ecently, both case-control and cohort studies have reported that maternal oral infections, as indexed by clinical measures of acute gingival infections (gingivitis) and chronic periodontal infections (periodontitis) may be an independent contributor to abnormal pregnancy outcomes, including preterm births, growth restriction, and preeclampsia. ${ }^{1-8}$ However, not all case-control reports support an association between these clinical conditions, ${ }^{9}$ raising the possibility of potential confounding of the association by established risk factors, such as smoking, or other factors that may underlie both conditions. Both gingivitis and periodontitis are relatively common concomitant clinical conditions among pregnant women, although prevalence estimates during pregnancy vary considerably (gingivitis 30-100\% and periodontitis 5-20\%). ${ }^{10}$ The potential mechanisms underlying the reported association between periodontal conditions and preterm delivery have not been established. It has been demonstrated in humans that periodontal pathogens within dental plaque are capable of invading host periodontal tissues, eliciting recurrent bacteremias, translocating to distant tissues, and activating the hepatic acute phase response, especially during periods of disease progression. ${ }^{11}$

Because periodontal health generally worsens during pregnancy, and when periodontal disease is present, it is characterized by periods of exacerbation and remission, it is possible that the onset of new disease or periodontal progression during pregnancy may pose greater risk to the pregnancy than just the 
antepartum identification of disease. In the current investigation, we explore the role of antepartum periodontal conditions, periodontal disease onset, and periodontal disease progression during pregnancy on preterm birth risk.

\section{MATERIALS AND METHODS}

The Oral Conditions and Pregnancy Study (OCAP) was a multidisciplinary, longitudinal examination of maternal oral health and pregnancy outcome. Institutional review board approval was granted to conduct the study, and all subjects provided informed written consent before enrollment. Detailed methodology reporting the study design, procedures for patient enrollment, inclusion and exclusion criteria, examiner calibration, clinical measurements and data collection methods, chart and medical record abstraction, biological sampling methods (maternal serum, vaginal and gingival), fetal cord blood processing, and covariate and confounder definitions has been described. ${ }^{13}$ The study enrolled subjects for a 42 -month period beginning in December of 1997.

Our primary hypothesis-that maternal antepartum periodontal disease status was a significant risk factor for preterm birth at less than 37 weeks of gestation-was the main outcome. Spontaneous preterm birth, defined as delivery after spontaneous preterm labor or spontaneous premature rupture of membranes, and preterm birth at less than 32 weeks of gestation were secondary outcomes examined. The Duke University Medical Center Institutional Review Board provided approval to conduct the study. Eligible women were identified at their first or second prenatal visit and enrolled before 26 weeks of gestation. Women were excluded from participation if they were less than 18 years of age and without a legal guardian, were at greater than 26 weeks of gestation at study enrollment, or had a multiple gestation, chronic hypertension, pregestational diabetes, heart murmur or heart valve disease, history of fenfluramine-phentermine use (unless a normal echocardiogram was documented), any medical condition requiring antibiotic prophylaxis for dental treatment, human immunodeficiency virus (HIV) infection, or if delivery was planned at another institution. A tracking system was used to record recruitment and enrollment in order to determine eligibility, enrollment, and attrition rates (Epi Info 6.0, Centers for Disease Control and Prevention, Atlanta, GA). Mothers who experienced spontaneous abortions, elective abortions, or intrauterine fetal demise were not included in these analyses

All pregnant women who were seen for routine obstetric care within the Duke Obstetrics Clinic were potentially eligible for participation and consented during the initial obstetric visits. Pregnant women received comprehensive, full-mouth, periodontal examinations before 26 weeks gestational age to estimate whether baseline periodontal status predicted abnormal pregnancy outcomes. Baseline exams occurred at a median of 15 weeks, with an interquartile range of 11-19 weeks gestational age. Repeat oral examinations were performed bedside in $87.4 \%$ (891) of mothers within 72 hours postpartum to estimate whether a worsening of periodontal disease during pregnancy also conferred risk. At enrollment, subjects received an obstetric examination for ultrasound dating and collection of vaginal microbial samples. All OCAP subjects provided demographic information, medical history, and concurrent exposure and behavioral information by interview and written questionnaires, both antepartum and postpartum. Mothers were followed through delivery and neonatal discharge to collect data about gestational age at delivery, birth weight, and neonatal outcomes. A total of 5,400 women were screened, with $65 \%$ ineligible. Of the 1,945 eligible women, $63 \%$ consented. Attrition of the initial 1,224 consenting women occurred because of further identification of exclusion criteria $(\mathrm{n}=35)$, study withdrawal $(\mathrm{n}=122)$, and transfer for delivery at other hospitals $(\mathrm{n}=47)$, leaving 1,020 subjects who participated these analyses.

Full-mouth antepartum and postpartum periodontal examination included assessments of plaque score, gingival indices, bleeding scores, probing depths, and attachment loss at 6 sites per tooth, as described. ${ }^{13}$ All probing measurements were rounded down to the nearest millimeter, and determinations of progression reflected site-specific changes over time. Maternal antepartum periodontal disease was defined as a 3-level categorical variable: health, mild disease, or moderate-severe disease, as described previously. ${ }^{2}$ Mothers with periodontal health were defined by these criteria: 1) all probing depths were $4 \mathrm{~mm}$ or less and 2) no 3-mm or 4-mm sites experienced bleeding on probing. On examination, $27.9 \%$ of the mothers exhibited periodontal health at enrollment and served as the referent group. The remaining mothers with antepartum gingivitis and periodontitis were divided into 2 groups. Those with the most disease, which would be described by American Academy of Periodontology guidelines as moderate-severe periodontal disease, had 15 or more sites with greater than 4-mm probing depths and represented $14.4 \%$ of OCAP mothers. The remaining $57.6 \%$ of mothers were described as having mild disease, which included the American Academy of Periodontology 
classifications of gingivitis and mild periodontal disease. The median (25th, 75th percentile) number of sites with 2-mm attachment loss were $0(0,3), 1(0,5)$, and $4(0,22)$ for health, mild disease, and moderatesevere disease, respectively. Similarly, the number of sites with bleeding on probing was $3(1,9), 32(18,54)$, and $104(56,144)$ for these 3 disease levels.

Periodontal progression (progressing gingivitis/ periodontitis) was determined by comparing sitespecific probing measurements between the antepartum and postpartum visits. Progression was defined as 4 or more sites with $2 \mathrm{~mm}$ or more of increasing probing depths at each site, with the postpartum probing depth being $4 \mathrm{~mm}$ or more. Otherwise, the women were classified as stable/not progressing. Although the time interval between the antepartum and postpartum examinations varied by gestational age at enrollment and delivery date, separate models were evaluated using rates of progression that tended to make the associations stronger for earlier gestational births, but these are not presented here so as to avoid the complexity of the variable definitions.

All dental examiners underwent training and calibration at the beginning of their participation and at 1-year intervals thereafter. ${ }^{13}$ Percentage agreement with a standard examiner within $1-\mathrm{mm}$ was greater than 96\%. Weighted kappas and intraclass correlation coefficients exceeded 0.9. Periodontal examiners were blinded with regard to obstetric outcomes.

Several variables were evaluated as possible risk factors, covariates, or confounders, including age at enrollment in 5-year increments, race (white/nonwhite), first birth, previous preterm delivery, smoking during current pregnancy, marital status, whether subjects were in either the Women, Infants, and Children Program (WIC) or food stamps assistance program, health insurance status, illicit drug use (patient reported use of marijuana, cocaine, or intravenous drug), alcohol consumption, and the presence of sexually transmitted disease (defined by Neisseria gonorrhoea and/or Chlamydia trachomatis testing) and chorioamnionitis (maternal temperature $>38^{\circ} \mathrm{C}$ and no other source of fever). The presence of bacterial vaginosis was based upon presentation of symptomatic vaginal discharge, vaginal $\mathrm{pH}$ of 4.5 or more, and wet mount identification of clue cells.

Gestational age was calculated by date of last menstrual period and considered confirmed if concordant within 10 days with a first- or second-trimester ultrasound examination. If no menstrual dates were available or if discordant by ultrasound dating by more than 10 days, ultrasound dating was used. Any mother who did not have ultrasound-confirmed dat- ing was excluded. Preterm birth was defined as live births at less than 37 weeks of gestation. Very preterm birth was defined as birth at less than 32 weeks of gestation. Spontaneous preterm or very preterm birth included only mothers with preterm rupture of membranes or preterm labor resulting in delivery at less than 37 or less than 32 weeks, respectively.

Bivariate analyses were performed for baseline characteristics, exposures, and behaviors of OCAP subjects using $\chi^{2}$ for dichotomous variables or $t$ tests for continuous variables for each outcome of interest. Logistic regression models were used to derive estimates of odds ratio, which were converted to relative risks and 95\% confidence intervals using an SAS macro (SAS Institute Inc, Cary, NC) for adjusted models. Certain traditional risk factors, such as smoking, history of preterm birth, age, and race, were always included as control variables in adjusted models irrespective of whether there was significant bivariate association or confounding. These were used to create risk models that would be comparable with other obstetric risk studies. Covariates were included in the multivariable models based upon a significant bivariate association with the outcome $(P \leq .05)$. Potential interactions of the covariates on the association between the main exposures and outcomes were evaluated with the intention of stratifying the model on that variable. However, no significant interactions were observed. Potential covariates that were not significantly associated with the outcome pairwise but with borderline significance $(.05<P<.2)$ were then added to the adjusted models to create fully adjusted models. These variables were retained in the fully adjusted models based upon either being a significant main effect in the model $(P<.05)$ or confounding the association between the periodontal exposure variables and the outcome by $5 \%$ or more. This process was used for models with outcomes of 37-week gestational age deliveries. However, because the number of cases was smaller for the 32-week gestational age models, backward selection procedures were used to reduce the number of variables in the model. Smoking was maintained as a control variable in all models. These procedures did not change the statistical significance or relative risk estimates for the main exposures. PROC Logistic (SAS Institute Inc) with the C statistic option was used to estimate the area under an receiver operator characteristic (ROC) curve. The effect of periodontal disease as an exposure was modeled using the 3-level disease variable, periodontal progression/nonprogression, or both. The $\chi^{2}$ for trend was used across the 3 periodontal disease levels for testing significant association with pregnancy outcome. 
Kaplan-Meier curves were generated for gestational age at delivery for 1,020 live births based upon 3-level antepartum periodontal status or gingivitis/ periodontal disease progression. Statistical significance between groups in this unadjusted survival function were determined by the Mantel Haenszel test.

\section{RESULTS}

The results of the bivariate analyses are shown in Table 1 . The preterm birth rate $(<37$ weeks of gestation) was $186(18.2 \%)$ of 1,020 . Preterm birth was significantly associated with maternal age, race, marital status, use of public assistance, insurance status, prior preterm birth, and clinical chorioamnionitis at delivery. The 169 women who either withdrew or delivered elsewhere did not significantly differ from the 1,020 study population with regard to baseline characteristics shown in Table $1 .^{13}$

Maternal periodontal health status at enrollment/ baseline/antepartum and active clinical disease pro-

Table 1. Baseline Characteristics, Exposures, and Behaviors of OCAP Subjects During Pregnancy for Term and Preterm Delivery Outcomes

\begin{tabular}{|c|c|c|c|}
\hline \multirow[b]{2}{*}{ Variable } & \multirow[b]{2}{*}{$\begin{array}{l}\text { All Mothers } \\
(n=1,020)\end{array}$} & \multicolumn{2}{|c|}{ Gestational Age Outcome } \\
\hline & & $\begin{array}{c}G A \geq 37 w k \\
(n=834 ; 81.8 \%)\end{array}$ & $\begin{array}{c}\mathrm{GA}<37 \text { wk } \\
(\mathrm{n}=186 ; 18.2 \%)\end{array}$ \\
\hline Maternal age $(y$, mean $\pm \mathrm{SD})$ & $28.2 \pm 6.6$ & $28.5 \pm 6.7$ & $26.7 \pm 6.0^{*}$ \\
\hline Maternal weight $(\mathrm{lb}$, mean $\pm \mathrm{SD})$ & $161.5 \pm 43.6$ & $160.7 \pm 43.7$ & $165.3 \pm 40.0$ \\
\hline African American & $471(46.2)$ & $346(73.5)$ & $125(26.5)^{*}$ \\
\hline White & $487(47.7)$ & $436(89.5)$ & $51(10.5)$ \\
\hline Other race & $62(6.1)$ & $52(83.9)$ & $10(16.1)$ \\
\hline Smoke & $162(15.8)$ & $124(76.5)$ & $38(23.5)$ \\
\hline No smoking & $856(84.2)$ & $710(82.8)$ & $148(17.2)$ \\
\hline Alcohol & $170(16.7)$ & $140(82.4)$ & $30(17.6)$ \\
\hline No alcohol & $850(83.3)$ & $694(81.6)$ & $156(18.4)$ \\
\hline Illicit drugs & $42(4.1)$ & $35(83.3)$ & $7(16.7)$ \\
\hline No drugs & $978(95.9)$ & $799(81.7)$ & $179(18.3)$ \\
\hline Not married & $497(48.7)$ & $388(78.1)$ & $109(21.9)^{\dagger}$ \\
\hline Married & $523(51.3)$ & $446(85.3)$ & $77(14.7)$ \\
\hline WIC or food stamp eligibility & $184(18.0)$ & $141(76.6)$ & $43(23.4)^{\ddagger}$ \\
\hline No WIC or food stamp eligibility & $836(82.0)$ & $693(82.9)$ & $143(17.1)$ \\
\hline No medical insurance & $536(52.5)$ & $418(78.0)$ & $118(22.0)^{\ddagger}$ \\
\hline Medical insurance & $484(47.5)$ & $416(86.0)$ & $68(14.0)$ \\
\hline First birth & $603(59.1)$ & $484(80.3)$ & $119(19.7)$ \\
\hline Multiparous & $417(40.9)$ & $350(83.9)$ & $67(16.1)$ \\
\hline Previous preterm delivery & $167(16.3)$ & $107(64.1)$ & $60(35.9)^{*}$ \\
\hline No previous preterm delivery & $853(83.7)$ & $727(85.2)$ & $126(14.8)$ \\
\hline STD & $146(14.4)$ & $117(80.1)$ & $29(19.9)$ \\
\hline No STD & $874(85.6)$ & $717(82.0)$ & $157(18.0)$ \\
\hline Chorioamnionitis & $13(1.2)$ & $3(23.1)$ & $10(76.9)^{*}$ \\
\hline No chorioamnionitis & $1007(98.8)$ & $831(82.5)$ & $176(17.5)$ \\
\hline Bacterial vaginosis & $77(7.5)$ & $63(81.8)$ & $14(18.2)$ \\
\hline No bacterial vaginosis & $943(92.5)$ & $771(81.8)$ & $172(18.2)$ \\
\hline Periodontal health & $285(27.9)$ & $253(88.8)$ & $32(11.2)$ \\
\hline Mild periodontal disease & $588(57.7)$ & $476(81.0)$ & $112(19.0)$ \\
\hline Moderate-severe periodontal disease & $147(14.4)$ & $105(71.4)$ & $42(28.6)^{*}$ \\
\hline Gingivitis/periodontitis stability & $658(73.8)$ & $558(84.8)$ & $100(15.2)$ \\
\hline Gingivitis/periodontitis progression & $233(26.2)$ & $175(75.1)$ & $58(24.9)^{*}$ \\
\hline
\end{tabular}

OCAP, Oral Conditions and Pregnancy; GA, gestational age; SD, standard deviation; WIC, Supplemental Food Program for Women, Infants, and Children; STD, sexually transmitted disease.

Values are expressed as mean \pm standard deviation or $\mathrm{n}(\%)$, as indicated.

Maternal weights and ages are shown at initial obstetric visit. Other baseline characteristics are categorized as present or absent during the index pregnancy except for race and antepartum periodontal disease, which is a 3-level variable as health, mild, or moderate severe. Smoking, alcohol, illicit drug use during current pregnancy was obtained by initial history, chart abstraction, and confirmed by postpartum interview. Periodontal gingivitis/periodontitis progression refers to changes in periodontal status comparing antepartum clinical parameters to postpartum on 891 mothers to reflect active disease progression as an exposure during current pregnancy. $P$ values reflect chi squared analyses for categorical variables. $P$ values for mean weights and age were determined by $t$ test.

$* P<.001$

$\dagger P<.005$.

$¥ P<.05$. 
gression are also shown in Table 1. The preterm birth rate was higher among women with moderate-severe periodontal disease $(28.6 \%)$ than among those with mild disease $(19.0 \%)$ or periodontal health $(11.2 \%)$, and women with clinical disease activity during pregnancy had higher rates than women with stable disease (24.9\% versus $15.2 \%)$. The unadjusted KaplanMeier survival function (pregnancy by gestational age) for 1,020 mothers stratified on baseline 3-level periodontal status is shown in Figure 1A. The rate of preterm delivery was slightly lower for the subset of $891(87.4 \%)$ subjects who had 2 oral examinations
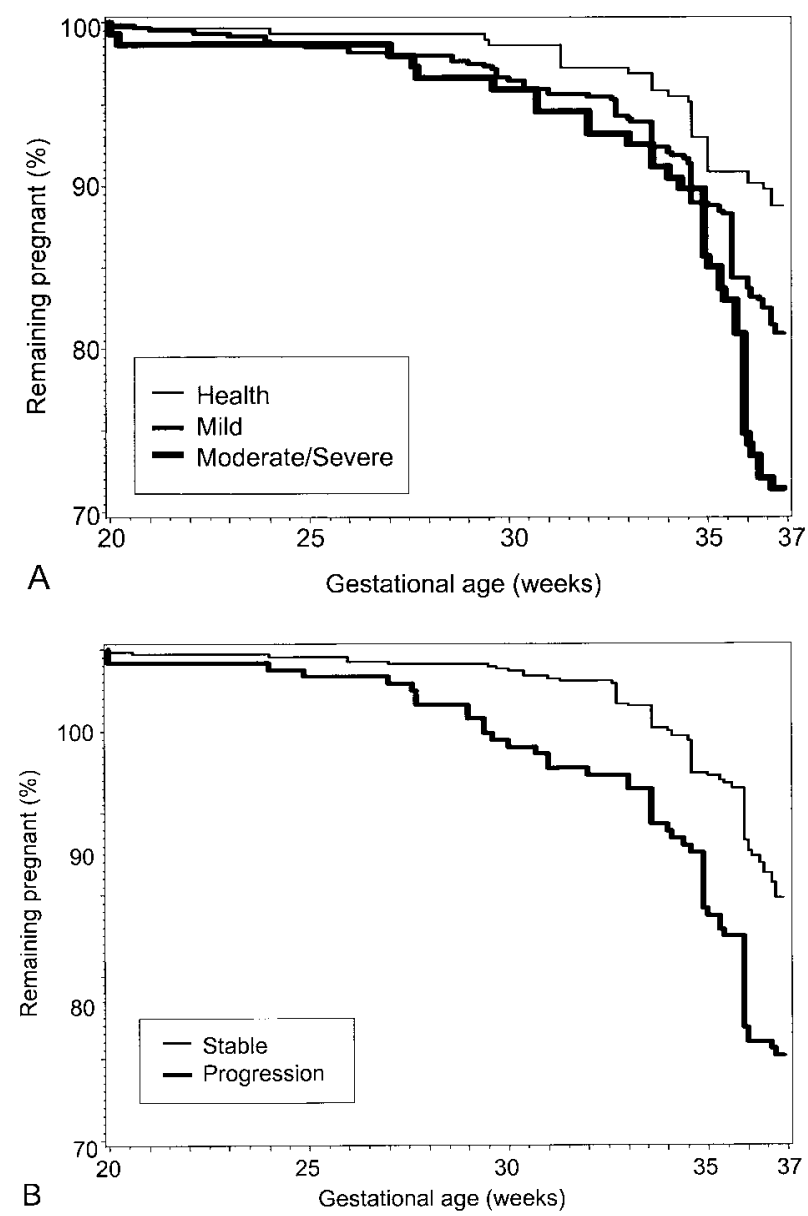

Fig. 1. A. Kaplan-Meier function for 1,020 pregnant women based upon 3-level antepartum periodontal disease status (health $[\mathrm{n}=285]$, mild disease [ $\mathrm{n}=588]$, or moderatesevere periodontal disease $[n=147])$. B. Kaplan Meier function for pregnancy-gestational age outcomes among 891 mothers with both antepartum and postpartum periodontal examination data available. Figure $1 \mathrm{~B}$ reflects mothers delivery outcomes for mothers with no gingivitis/ periodontitis progression ( $n=233$ ) or with active gingivitis/ periodontitis progression $(\mathrm{n}=658)$.

Offenbacher. Periodontitis Increases Very Preterm Births. Obstet Gynecol 2005 compared with those with only the antepartum examination (17.7\% versus $21.7 \%$, respectively) but did not represent a statistically significant sampling bias $(P=.3)$.

The Kaplan-Meier curve for mothers stratified by active clinical periodontal progression or nonprogression during the current pregnancy is shown in Figure 1B. Active progression during pregnancy was associated with an increased risk for preterm birth $(P<$ $.001)$. In contrast to the effect associated with antepartum periodontal disease that demonstrates large differences near term (35-37 weeks of gestation) for mild and moderate-severe periodontal disease (Fig. 1A), maternal progression during pregnancy has a trend at earlier gestational ages demonstrating differences in the 2 survival curves separating before 32 weeks (Fig. $1 \mathrm{~B})$, suggesting that periodontal disease progression is more strongly associated with very preterm births.

The risk factor-adjusted relative risk $(\mathrm{RR})$ models for preterm birth and spontaneous preterm birth according to antepartum periodontal status using the 3-level periodontal disease variable as the main exposure are shown in Table 2. Compared with mothers with periodontal health, the relative risk for both preterm delivery and spontaneous preterm delivery was significantly elevated for mothers with moderatesevere periodontal disease (preterm birth adjusted RR 1.6, 95\% confidence interval [CI] 1.1-2.3; spontaneous preterm birth adjusted RR 2.0, 95\% CI 1.2-3.2). These 2 models were adjusted for age, race, first birth, previous preterm delivery, smoking during pregnancy, marital status, Supplemental Food Program for Women, Infants, and Children (WIC) or food stamps, health insurance status, and the presence of chorioamnionitis.

We then explored the effect of maternal periodontal disease progression on preterm birth risk (Table 3). Active periodontal disease progression was not associated with significant risk of preterm birth at less than 37 weeks of gestation (data not shown). However, the unadjusted rate of very preterm delivery was $6.4 \%$ among women with periodontal disease progression-significantly less than the $1.8 \%$ rate among mothers without disease progression $(P<.001$ by $\left.\chi^{2}\right)$. Women with active clinical periodontal disease progression were at significant risk for delivery at gestational age less than 32 weeks, with an adjusted risk ratio of 2.4 (95\% CI 1.1-5.2), after adjusting for race, first birth, previous preterm delivery, smoking, and chorioamnionitis. Inclusion of antepartum periodontal status in the predictive model of birth at gestation age less than 32 weeks did not significantly impact the risk associated with active disease progression (RR 2.5, 95\% CI 1.1-5.4), and antepartum peri- 
Table 2. Risk Factor-Adjusted Relative Risk for Preterm Births According to Antepartum Maternal Periodontal Disease Status

\begin{tabular}{lcc}
\hline Variable & Preterm* & $\begin{array}{c}\text { Spontaneous } \\
\text { Preterm* }\end{array}$ \\
\hline Periodontal health & 1.0 (referent) & 1.0 (referent) \\
Mild periodontal disease & $1.2(0.9-1.7)$ & $1.5(<1.0-2.2)$ \\
Moderate-severe periodontal disease & $1.6(1.1-2.3)^{\dagger}$ & $2.0(1.2-3.2)^{\ddagger}$ \\
Age (5-y increment) & $0.9(0.8-1.04)$ & $0.9(0.8-1.04)$ \\
Race (white) & $0.5(0.4-0.7)$ & $0.5(0.4-0.8)$ \\
First birth & $0.9(0.6-1.2)$ & $0.9(0.6-1.2)$ \\
Previous preterm & $2.0(1.4-2.7)$ & $2.4(1.7-3.4)$ \\
Smoking & $1.2(0.9-1.6)$ & $1.2(0.8-1.7)$ \\
Married & $1.1(0.8-1.5)$ & $1.2(0.8-1.7)$ \\
WIC or food stamps & $1.0(0.7-1.4)$ & $1.1(0.8-1.6)$ \\
Insurance & $1.1(0.8-1.6)$ & $1.2(0.8-1.8)$ \\
Chorioamnionitis & $3.1(1.7-5.4)$ & $4.3(2.2-8.4)$ \\
\hline
\end{tabular}

WIC, Supplemental Food Program for Women, Infants, and Children.

Data are expressed as relative risk (95\% confidence interval).

* Predictive risk models for preterm births (gestational age $<37$ weeks, 187 cases) and spontaneous preterm births (spontaneous gestational age $<37$ weeks, 170 cases).

$\dagger P=.02$ for tests of trend across increasing periodontal disease severity.

$¥ P=.003$ for tests of trend across increasing periodontal disease severity.

Table 3. Risk Factor-Adjusted Relative Risk for Very Preterm Births* for Mothers With Periodontal Disease Progression During Pregnancy

\begin{tabular}{lc}
\hline Variable & $\begin{array}{c}\text { Relative Risk } \\
\text { (95\% Confidence } \\
\text { Interval) }\end{array}$ \\
\hline Periodontal disease, no progression & 1.0 (referent) \\
Periodontal disease, progression & $2.4(1.1-5.2)$ \\
Race (white) & $0.1(0.02-0.5)$ \\
First birth & $0.3(0.1-0.9)$ \\
Previous preterm & $3.4(1.3-9.1)$ \\
Smoking & $0.6(0.2-2.1)$ \\
Chorioamnionitis & $9.5(3.7-24.2)$ \\
\hline
\end{tabular}

*Very preterm births: gestational age $<32$ weeks; $\mathrm{n}=27$.

odontal status is not included in the model shown in Table 3 . All but 2 of the very preterm births were spontaneous deliveries, limiting our ability to stratify association of active clinical periodontal disease progression based on type of very preterm birth.

\section{DISCUSSION}

Our findings demonstrate that maternal periodontal disease, identified either early in pregnancy or progressing during pregnancy, is a risk factor for preterm and very preterm birth, respectively, independent of other risk factors. Antepartum moderate-severe periodontal disease increased the risk for spontaneous preterm birth 2-fold in fully adjusted models, and the effect of active periodontal disease on very preterm delivery was even larger (adjusted RR 2.4, 95\% CI 1.1-5.2).
Periodontal disease is a chronic infection, and when present, the natural history of the disease is characterized by episodic periods of quiescence and progression ${ }^{14}$ Furthermore, pregnancy increases the onset of new periodontal disease. ${ }^{10}$ These patterns would suggest that, if the preexisting periodontal disease becomes active during the pregnancy, it may pose a significant concomitant infectious or inflammatory exposure during the current pregnancy, as would the onset of new disease. In this context the OCAP investigation provides novel information to demonstrate that active clinical periodontal disease progression during pregnancy conveys significant risk for very preterm birth, independently of preexisting periodontal disease.

The results of our study should be considered in light of previously published data on maternal periodontal disease and preterm birth. ${ }^{15}$ The magnitude of the association between maternal periodontal disease and preterm birth in our study is lower than that reported by Jeffcoat et al. ${ }^{5}$ In a prospective study of 1,313 pregnant women, Jeffcoat and colleagues reported that severe periodontal disease is associated with an odds ratio of 5.28 (95\% CI 2.05-13.6) for preterm birth at less than 37 weeks and an odds ratio of 7.07 (95\% CI 1.7-27.4) for preterm birth at less than 32 weeks, adjusting for age, race, smoking, and parity. The differences in magnitude of association between our study and the data of Jeffcoat et al may be due to differences in baseline maternal oral health between our study cohort and the cohort in the Jeffcoat et al Alabama study population because the 
women in Alabama had significantly worse oral health than the women in our cohort. However, both studies show a biological gradient effect, in that more severe periodontal disease was associated with a stronger risk for earlier preterm birth.

A recent prospective study of 3,738 births conducted in London by Moore and colleagues ${ }^{16}$ failed to demonstrate an association between periodontal disease severity and preterm births or low birth weight (LBW) infants. However, this investigation did note a trend for an increase in second-trimester fetal loss $(P=.057$, odds ratio 5.5) in mothers with severe disease. A similar negative finding by Davenport et al, ${ }^{9}$ also conducted in London, suggests that perhaps not all maternal populations with periodontal disease are at increased risk for pregnancy complications. Both the Alabama and OCAP studies have a greater proportion of African Americans, who historically are at significantly greater risk for both periodontal disease (approximately 2 -fold), ${ }^{17}$ preterm births, and LBW infants. ${ }^{18}$ Although African Americans represent $21.0 \%$ of the total North Carolina population of women of childbearing age, the OCAP population was almost half African American, and this group accounted for $67 \%$ of all preterm births. Thus, OCAP subjects reflect state and national patterns of increased risk for prematurity among African Americans as compared with whites (2-fold adjusted RR in OCAP, Table 1). Considering the high concordance of both periodontal disease and pregnancy complications among African Americans, it is not unreasonable to consider that periodontal disease may, in part, contribute to this racial disparity, ie, the high prevalence of abnormal pregnancy outcomes among African Americans.

Smoking during pregnancy was not a significant risk factor for preterm birth in our study. Only 15.8\% of women reported smoking, which is identical to the average reported rate among pregnant women in North Carolina in $1996(15.8 \%) \cdot{ }^{19,20}$ There was no significant difference in reported smoking rates between African Americans and whites (17.8\% versus $13.8 \%$, respectively). There were differences in smoking among women stratified by periodontal disease category (healthy mothers $6.7 \%$, mild disease $17.5 \%$, and moderate-severe disease $27.2 \% ; P<.001$ ). It is possible that reported smoking rates underestimate the true rate of smoking, but results from anonymous cotinine levels determined at our institution suggest that this is not the case (data were obtained from a different pregnancy outcome study and are not shown). Nonetheless, as a traditional obstetric risk factor, tobacco usage was included as a dichotomous control variable in all adjusted models. Other measures of tobacco exposure were tested in predictive models using pack-years or current levels of smoking, and these variables did not significantly modify the relationship between periodontal disease and preterm or very preterm births (data not shown).

This investigation sought to control for the potential confounding of social domain variables. The severity of periodontal disease has been weakly associated with poor oral hygiene and moderately associated with lower education and lower income in epidemiological studies. ${ }^{17}$ Lower socioeconomic status, as indexed by education, income, and access to care, has also been associated with increased risk for abnormal pregnancy outcomes. ${ }^{18}$ In OCAP, 18\% of subjects were either on food stamps or in WIC program, almost half were unmarried, and over half lacked medical insurance. These 3 variables were chosen to represent social domain factors. Although we collected information on a larger range of social domain variables, including household income, level of education, family support, and abuse, none of these were significant in modifying the relationship between periodontitis and abnormal pregnancy outcomes or were not significant as main effect variables when including these 3 variables in models.

The potential for maternal periodontal disease, which is a chronic oral infection, serving as a surrogate marker for other maternal infections is of particular interest. Chorioamnionitis was strongly associated with preterm and very preterm birth among OCAP mothers, but a diagnosis of sexually transmitted diseases or bacterial vaginitis was not significantly associated with increased risk. In a case-control study by Goepfert et al, ${ }^{21}$ severe periodontal disease was more common among the spontaneous preterm birth group, but neither positive placental or umbilical cord cultures, histological chorioamnionitis, nor funisitis were associated with more severe periodontitis. Although more studies are needed, the data thus far indicate that the effects of periodontal disease on preterm birth appear to be independent of vaginal and reproductive track infections, suggesting that periodontal disease presents an oral route of infectious burden on the maternal-fetal unit that is distinct from the reproductive tract.

It should also be considered that the presence or progression of maternal periodontal disease may reflect an intrinsic inflammatory or innate immunity trait that places the mother at risk for not only more severe periodontal disease but also at greater risk for the common terminal biochemical inflammatory cascade associated with abnormal pregnancy outcomes, 
as described by Romero and Mazor ${ }^{22}$ and Williams et al. ${ }^{23}$ For example, it is not unreasonable to suggest that mothers with an underlying propensity for excess secretion of inflammatory mediators and cytokines, such as prostaglandin $\mathrm{E}_{2}$, interleukin (IL)-6, or IL- $1 \beta$, may be simultaneously at risk for both periodontal disease and abnormal pregnancy outcomes. The presence of an underlying abnormal inflammatory trait for the mother or fetus may worsen the clinical impact of a potential infectious exposure during pregnancy, such as periodontal disease, as in this case. Thus, this study can not rule out the possibility that the observed association is not, in part, attributable to an underlying inflammatory or other common risk factor that places mothers at risk for both conditions.

Active maternal periodontal disease appears to have the most deleterious effect early in pregnancy. Although in North Carolina, births that occur between 35 and 37 weeks of gestation represent $58 \%$ of all preterm births, ${ }^{19}$ the mortality rate for neonates at gestational age less than 35 weeks is 11.4-fold higher than that at gestational age greater than 35 weeks, and survivors are at higher risk for significant morbidity, including paraventricular leukomalacia and periventricular hemorrhage, cerebral palsy, and chronic lung disease of the newborn. ${ }^{24}$ The findings from OCAP indicate that maternal periodontal disease progression during pregnancy may, in part, contribute to deliveries at less than 32 weeks of gestation and that maternal periodontal disease progression merits further consideration as a potential risk factor for neonatal morbidity and mortality.

\section{REFERENCES}

1. Offenbacher S, Katz V, Fertik G, Collins J, Boyd D, Maynor G, et al. Periodontal infection as a possible risk factor for preterm low birth weight. J Periodontol 1996; 67 suppl:S1103-13.

2. Offenbacher S, Lieff S, Boggess KA, Murtha AP, Madianos $\mathrm{PN}$, Champagne CM, et al. Maternal periodontitis and prematurity. Part I. Obstetric outcome of prematurity and growth restriction. Ann Periodontol 2001;6:164-74.

3. Madianos PN, Lieff S, Murtha AP, Boggess KA, Auten RL Jr, Beck JD, et al. Maternal periodontitis and prematurity: Part II. Maternal infection and fetal exposure. Ann Periodontol 2001; 6:175-82.

4. Dasanayake AP. Poor periodontal health of the pregnant woman as a risk factor for low birth weight. Ann Periodontol 1998;3:206-12.

5. Jeffcoat MK, Geurs NC, Reddy MS, Cliver SP, Goldenberg RL, Hauth JC. Periodontal infection and preterm birth: results of a prospective study. J Am Dent Assoc 2001;132:875-80.

6. Lopez NJ, Smith PC, Gutierrez J. Higher risk of preterm birth and low birth weight in women with periodontal disease. J Dent Res 2002;81:58-63.
7. Romero BC, Chiquito CS, Elejalde LE, Bernardoni CB. Relationship between periodontal disease in pregnant women and the nutritional condition of their newborns. J Periodontol 2002;73:1177-83.

8. Boggess KA, Lieff S, Murtha AP, Moss K, Beck J, Offenbacher $\mathrm{S}$. Maternal periodontal disease is associated with an increased risk for preeclampsia. Obstet Gynecol 2003;101:227-31.

9. Davenport ES, Williams CE, Sterne JA, Murad S, Sivapathasundram V, Curtis MA. Maternal periodontal disease and preterm low birthweight: case-control study. J Dent Res 2002; 81:313-8.

10. Laine MA. Effect of pregnancy on periodontal and dental health. Acta Odontol Scand 2002;60:257-64.

11. Champagne CM, Madianos PN, Lieff S, Murtha AP, BeckJD, Offenbacher S. Periodontal medicine: emerging concepts in pregnancy outcomes. J Int Acad Periodontal 2000;2:9-13.

12. Lin D, Smith MA, Elter J, Champagne C, Downey CL, Beck J, et al. Porphyromonas gingivalis infection in pregnant mice is associated with placental dissemination, an increase in the placental Th1/Th2 cytokine ratio, and fetal growth restriction. Infect Immun 2003;71:5163-8.

13. Lieff S, Boggess KA, Murtha AP, Jared H, Madianos PN, Moss $\mathrm{K}$, et al. The oral conditions and pregnancy study: periodontal status of a cohort of pregnant women. J Periodontol 2004;75: 116-26.

14. Beck JD, Offenbacher S. The association between periodontal diseases and cardiovascular diseases: a state-of-the-science review. Ann Periodontol 2001;6:9-15.

15. Scannapieco FA, Bush RB, Paju S. Periodontal disease as a risk factor for adverse pregnancy outcomes: a systematic review. Ann Periodontol 2003;8:70-8.

16. Moore S, Ide M, Coward PY, Randhawa M, Borkowska E, Baylis R, et al. A prospective study to investigate the relationship between periodontal disease and adverse pregnancy outcome. Br Dent J 2004;197:251-8.

17. Papapanou PN. Periodontal diseases: epidemiology. Ann Periodontol 1996;1:1-36.

18. Goldenberg RL, Hauth JC, Andrews WW. Intrauterine infection and preterm delivery. N Engl J Med 2000;342:1500-7.

19. North Carolina State Center for Health Statistics. Final mortality data. NC. 1999-2001. Available at: http://www.schs.state.nc.us/ SCHS/. Retrieved November 16, 2005.

20. Centers for Disease Control and Prevention. Smoking during pregnancy - United States, 1990-2002. MMRW Morb Mortal Wkly Rep 2004;53:911-5.

21. Goepfert AR, Jeffcoat MK, Andrews WW, Faye-Petersen O, Cliver SP, Goldenberg RL, et al. Periodontal disease and upper genital tract inflammation in early spontaneous preterm birth. Obstet Gynecol 2004;104:777-83.

22. Romero R, Mazor M. Infection and preterm labor. Clin Obstet Gynecol 1988;31:553-84.

23. Williams CE, Davenport ES, Sterne JA, Sivapathasundaram V, Fearne JM, Curtis MA. Mechanisms of risk in preterm lowbirthweight infants. Periodontol 2000 2000;23:142-50.

24. Leviton A, Paneth N, Reuss ML, Susser M, Allred EN, Dammann O, et al. Maternal infection, fetal inflammatory response, and brain damage in very low birth weight infants. Developmental Epidemiology Network Investigators. Pediatr Res 1999;46:566-75. 